\title{
Time resolved dynamic micro-CT imaging of food products in the lab
}

Frederik Coppens $^{1}$, Wesley De Boever ${ }^{2}$ and Jan Dewanckele ${ }^{1}$

${ }^{1}$ TESCAN, Belgium, ${ }^{2}$ Tescan, Belgium

The use of X-ray micro-CT to study the microstructure of food products has been well established over the past decades. In recent years, there is a growing trend towards in-situ and time resolved imaging. Advances in temporal resolution of X-ray CT systems make imaging of fast dynamic processes in the lab possible, where previously only synchrotron facilities allowed this. With TESCAN micro-CT systems temporal resolutions below 10 seconds can now be attained on a routine basis by using a combination of hard- and software designed specifically with this application in mind.

By means of application examples we discuss the essential concepts starting with micro-CT hardware selection and acquisition strategies. This is closely tied to the integration of in-situ devices both on hardas software level. Finally, dynamic imaging offers both unique advantages and poses challenges for reconstruction, visualization and analysis of 4D data.

When evaluating the processing conditions of solid foams such as cakes and bread, the integration of insitu devices is challenging. First, we present differences in porosity evolution between sourdough and modern bread baking during the leavening process. We move further with the baking process of muffins, where the ultimate macrostructure is the result of a complex process of bubble growth with contributions both at micro- as macroscopic level. Next, we showcase the influence of mixing on foam structure of whipped cream.

In the evaluation of product performance dynamic micro-CT can also play a complementary role to other technologies. We have a look at the evolution of the foam head of a strong Belgian ale. The structure and evolution of this fragile, highly aerated liquid foam has strong implications on both the sensory as the aesthetic quality of the product. Mechanical properties can play an important role in the sensory experience for a certain food product, which has a major influence on the perceived quality. The integration of an insitu three point bending stage allows to evaluate the mechanical properties during imaging, we show this for hard sweet cookies.

Reference

https://doi.org/10.1111/jmi.12879 